\title{
A Proposal for Introducing Quantum Physics in the Footsteps of Einstein ${ }^{\dagger}$
}

\author{
Marco Di Mauro ${ }^{1, *(\mathbb{D}}$, Salvatore Esposito ${ }^{2}$ and Adele Naddeo ${ }^{2}$ () \\ 1 Università di Salerno, Via Giovanni Paolo II, 84084 Fisciano, SA, Italy \\ 2 INFN Sezione di Napoli, Via Cinthia, 80126 Naples, Italy; salvatore.esposito@na.infn.it (S.E.); \\ anaddeo@na.infn.it (A.N.) \\ * Correspondence: madimauro@unisa.it; Tel.: +39-347-6541824 \\ + Presented at the 1st Electronic Conference on Universe, 22-28 February 2021; Available online: \\ https:/ / ecu2021.sciforum.net/.
}

\section{check for}

updates

Citation: Di Mauro, M.; Esposito, S.; Naddeo, A. A Proposal for Introducing Quantum Physics in the Footsteps of Einstein. Phys. Sci. Forum 2021, 2, 30. https://doi.org/ 10.3390/ECU2021-09320

Academic Editor: Magdalena Kersting

Published: 22 February 2021

Publisher's Note: MDPI stays neutral with regard to jurisdictional claims in published maps and institutional affiliations.

Copyright: (c) 2021 by the authors. Licensee MDPI, Basel, Switzerland. This article is an open access article distributed under the terms and conditions of the Creative Commons Attribution (CC BY) license (https:// creativecommons.org/licenses/by/ $4.0 /)$.

\begin{abstract}
We formulate a didactic proposal for introducing some fundamental concepts of quantum physics to advanced high school students, and to their teachers. The inspiration comes from some of the fundamental papers about the subject by Albert Einstein, in which many of these concepts, for example light quanta, wave-particle duality, and probability, were introduced for the first time, in a characteristically illuminating way. The proposal can be supplemented by a discussion of elementary tools of statistical physics, which are needed at some point. Preliminary results, both with students and teachers, are very promising.
\end{abstract}

Keywords: physics teaching; history of physics; quantum mechanics

\section{Introduction}

Nowadays physics teachers and researchers are greatly challenged by the need to develop educational tracks aimed at introducing advanced high school students to the main concepts of quantum theory [1], in order to engage them with modern physics and to stimulate their interest towards science and technology. This is made more urgent by the fact that many high school teachers may lack a proper advanced physics education (for example if their major was in mathematics, as often happens in Italy). Hence, teachers themselves should benefit from appropriately designed educational paths. It has been repeatedly emphasized that the history of physics can play an important role in teaching, in multiple ways (see [2]). One of the possibilities, especially for modern physics, is taking inspiration from the reading of original texts by the founders of the subject. This is especially the case for quantum physics, the teaching of which is very often developed by following a historical path. Indeed, the typical teaching-learning sequence for quantum physics starts from Planck's formula for black body radiation, and gradually introduces-roughly in the same order in which they were discovered - the main physical concepts that in the end will be subsumed in the full theory of quantum mechanics. Usual textbook treatments of elementary quantum physics employ a rather standardized choice of topics, of course at different depths, depending on their target, be it undergraduate or high school students. Examples include [3-6]. While we have no objection towards this way of developing the subject, we recognize that history is far more complex and rich than any didactic presentation. While it is of course natural that textbooks which are not history manuals simplify the historical development, it may still be the case that some additional insight could be gained by looking at usually neglected parts of history. In the present paper, we suggest that such insight can be found in some of Einstein's fundamental works in quantum physics. In fact, as is well known by historians, many of the fundamental concepts that nowadays are at the core of quantum mechanics, have been introduced in some paper by Einstein [7]. Despite this, most of these works are often ignored in teaching. For example, after dis- 
cussing Planck's radiation formula and its justification in terms of energy quantization, it is customary to go on immediately to the explanation of the photoelectric effect in terms of light quanta. This contribution, revolutionary as it is, is often the only one by Einstein that is mentioned. The deep difference between Planck's hypothesis of energy quantization and Einstein's light quanta is often not emphasized. Nor is there any mention of Einstein's statistical reasoning in order to arrive at the latter [8]. Additionally, the great conceptual leap involved in going from the energy quantization or light quanta hypotheses, which are statistical in nature, to the application to the photoelectric effect, in which individual quanta are involved, is often not stressed enough. After this, traditional courses go straight to discussing Bohr's atomic model, and then to De Broglie's hypothesis of matter waves. The pivotal introduction by Einstein of the concept of wave-particle duality for light (later extended to matter), again by a statistical reasoning applied to the black body radiation formula [9], which shows that both the wave and the particle nature of light are necessary for accounting for the observed spectrum, is usually neglected. Going on, while Einstein's modern derivation of the Planck formula in terms of atomic transitions [10] is sometimes treated (especially in view of its important application to lasers), the really revolutionary part, namely the intrinsic probabilistic and causality violating nature of the process of spontaneous emission, is not properly emphasized. Yet, this was the first appearance of probably the most mind-blowing characteristics of quantum mechanics. Notable exceptions in the literature, which do discuss one of more of these aspects, include [11,12], as well as explicitly historically oriented works such as $[13,14]$. However, these are quite advanced works, while typical high school books completely neglect these developments. However, we believe that also advanced high school students should benefit from a discussion of the above-mentioned topics, if of course they are presented in a suitably simple way. The same is true for teachers, with the advantage of their wider mathematical and physical background, which allows a deeper treatment. An important feature of many of Einstein's papers is that they are uniformly models of clarity and clear physical thinking. Additionally, unlike many papers by Einstein's contemporaries, these works turn out to be very readable and enlightening, also for modern readers. Moreover, all of Einstein's papers up to 1927 are freely available in English translation on the Internet [15], and translations in many other languages are often republished. These papers, full of marvellous insight, deep philosophical discussions, and examples of how great science is done, can therefore constitute wonderful and stimulating reading for high school teachers and, in a suitable selection, for pupils.

Inspired by the above considerations, we have extracted a didactic path to quantum physics from the papers by Einstein we have cited. We have tried to stay as close as possible to Einstein's original reasonings, while trying to simplify the mathematics whenever possible, so that nothing more complicated than elementary integral calculus is used, but without losing physical insight. This makes the derivations affordable by advanced high school students. Moreover, modern notation is used throughout. Emphasis is on clarity rather than on mathematical rigor.

\section{The Track}

In this section, we give some details on the didactic track we developed. In order to be concise, those parts of it which rely on standard material which is readily available in the literature are only sketched. It is assumed that students have studied the standard curriculum of classical physics, up to electromagnetic waves, and including the kinetic theory of gases. Moreover, the ability to perform simple integrals is taken for granted. Students in the second half of their last year of high school should therefore be prepared to undertake this path. 


\subsection{Step 1: Cavity Radiation and Planck's Law}

As do most teaching-learning sequences in quantum physics, ours begins with Planck's law for the energy density of radiation in a cavity at thermal equilibrium:

$$
u(v, T)=\frac{8 \pi h v^{3}}{c^{3}} \frac{1}{e^{\frac{h v}{k_{B} T}}-1}
$$

This law can be introduced in a phenomenological way as a function which fits experimental data on thermal radiation (this is actually how Planck first introduced it), and it can be treated in a standard way. A heuristic explanation of why energy quantization allows to get Planck's formula can be given in terms of how the former affects the principle of equipartition of energy which, when applied to electromagnetic waves, would give the unacceptable Rayleigh-Jeans (RJ for short) law. Several aspects need to be emphasized, in order for what follows to be clearer. In particular, the universality of black body radiation, and hence of the constants which appear in it, specifically Planck's constant $h$. Then, it is important to see how the limit $\frac{h v}{k_{B} T} \ll 1$, in which energy quanta are much smaller than the average thermal energy, which is called the classical limit (in this limit $h$ cancels out), gives back the Rayleigh-Jeans law, while the opposite limit $\frac{h v}{k_{B} T} \gg 1$, which may be dubbed the "extremely quantum limit", gives a limiting form of the Planck law, which is the Wien law:

$$
u(v, T)=\frac{8 \pi h v^{3}}{c^{3}} e^{-\frac{h v}{k_{B} T}}
$$

This is an important step, as Einstein introduced light quanta by studying the latter law, which is valid precisely in the regime where the quantum nature of light is most evident.

\subsection{Step 2: Einstein 1905: Light Quanta}

In his 1905 paper [8], Einstein computes in an ingenious way the entropy density associated with thermal radiation described by Wien's distribution (2). The computation is quite easy, as it involves standard thermodynamics and the integration of a logarithmic function. The result can be used to obtain the entropy variation under an isothermal and adiabatic expansion from a volume $V_{0}$ to a volume $V$. This is given by:

$$
S(V)-S\left(V_{0}\right)=k_{B} \ln \left(\frac{V}{V_{0}}\right)^{\frac{E}{h v}}
$$

and is valid separately for each frequency component of the radiation. This can be compared with the entropy variation associated with the Joule expansion (that is, a free expansion which is both adiabatic and isothermal) of the ideal gas, which is as well known $S(V)-S\left(V_{0}\right)=k_{B} \ln \left(\frac{V}{V_{0}}\right)^{N}$, where $N$ is the number of molecules making up the gas. This expression can be easily and instructively derived from Boltzmann's statistical formula for entropy. It is striking that the two expressions are exactly the same, provided one identifies

$$
\frac{E}{h v}=N \quad \text { or } \quad E=N h v
$$

Einstein's bold interpretation of the above identification is that radiation behaves statistically as if it were made up of $N$ independent "quanta", each of which has energy given by Planck' expression $h v$, so that the total energy of the radiation is just the sum of the energy of individual quanta, which behave just as the molecules of an ideal gas. It is important to emphasize that this statement is much more revolutionary than Planck's hypothesis, according to which only the exchanges of energy between the radiation and the walls of the cavity are discrete. Here, it is instead argued that the radiation itself is quantized, at least in the limit where quantum effects dominate, and Wien's law is valid. In 
doing this, Einstein for the first time used statistical physics in a peculiar and illuminating way. In fact, he used the expression of the entropy coming from thermodynamics to infer the statistical behaviour of radiation, while usually the opposite is done.

\subsection{Step 3: Some Applications}

At this point, it is useful to consider some applications of the above result. These applications actually go one step further, as they assume that not only the statistical behavior of the radiation is given by its corpuscular nature, but that the physical effects of individual quanta can be measured. Hence, they show that quantization of radiation is a fundamental feature of Nature. In his paper [8], Einstein considers three applications, one of which is the famous one to the photoelectric effect, which of course must be included in the track. It can be treated in a standard way (e.g., as in [3]). We have also found that another of these applications, which is usually not treated, namely the explanation of Stokes' rule for fluorescence, can be useful and instructive. Such rule, in fact, states simply that, when a fluorescent material absorbs light, it re-emits it with a lower frequency. A well-known effect is the violet glow of white clothes, which are exposed to ultraviolet light. (This is usually well known by students, especially those who like going to the disco.) This rule is readily understandable in terms of light quanta. Namely, fluorescent materials absorb light quanta, whose energy is $\varepsilon=h v$, and re-emit them. As part of the energy is absorbed, light quanta are re-emitted with a lower energy $\varepsilon^{\prime}<\varepsilon$. However, $\varepsilon^{\prime}=h v^{\prime}$, hence $v^{\prime}<v$, which is precisely Stokes' rule. The third application concerns ionization by UV light quanta, and it can be considered as well.

\subsection{Step 4. Einstein 1909: Wave-Particle Duality}

Going back to the full Planck distribution, an interesting consideration can be made. In the classical limit, it reduces to the RJ distribution, which can be obtained by applying equipartition of energy to electromagnetic waves. On the other hand, in the "most quantum" limit, it reduces to the Wien distribution which, as we just saw, leads to considering radiation as an ideal gas of non-interacting quanta. However, the full Planck distribution contains both these limits. This means that, while at extreme values of the frequencies, an entirely wave or an entirely particle picture works well; for the full distribution both aspects are necessary. Giving a quantitative foundation to this observation was what Einstein did in 1909 [9]. In that paper, Einstein brilliantly applied a statistical reasoning to the full Planck radiation law (1). This time, he focused on quadratic fluctuations of the energy, which can be computed by using elementary statistical physics methods, namely Boltzmann's distribution of energy. At this point a slight detour may be advisable, as this topic is not usually known by students. The result of the computation, which once again does not involve difficult mathematical steps, is

$$
(\Delta E)^{2}=\left(h v u+\frac{c^{3}}{8 \pi} \frac{u^{2}}{v^{2}}\right) V d v
$$

Again, this result is separately valid for all frequencies. The first term has exactly the same form of what one could get from an ideal gas, when one restricts to molecules having all the same energy $\varepsilon=h v$ (the analog of monochromatic radiation), which is $(\Delta E)^{2}=\varepsilon^{2} N$, where $N=E / h v$ is the average number of molecules, and it is the only term one gets when one considers the Wien distribution in place of the Planck version. The other term is what one would obtain by considering waves (the fluctuations are quadratic in the energy density due to interference effects), and it is the only contribution if the RJ law is used. Equation (5) shows that the Planck distribution involves both a particle contribution and a wave contribution. Einstein's genius revealed itself in the choice of the right quantity, in which the two contributions are unentangled, and appear as a simple sum. This result, Einstein concluded, was leading to (in his words) "a theory of light which can be interpreted as a kind of fusion of the wave and the emission [i.e., the corpuscular] theory." 


\subsection{Step 5. Interlude: The Bohr Atom}

Einstein's next breakthrough paper, which we are discussing in the next subsection, was inspired by Bohr's atomic model. Therefore, this model has to be properly treated. Again, this can be done in the usual way [3]. The important result for what follows is that, according to that model, electrons in atoms and molecules (not only in the hydrogen atom) can only occupy a set of stationary states $Z_{1}, Z_{2}$, etc. with discrete energies $E_{1}, E_{2}$, etc., and transitions between these states can occur. According to the Bohr theory postulates, in the transition between the $m$-th state and the $n$-th state, with $E_{m}>E_{n}$, if the electron goes from $Z_{m}$ to $Z_{n}$, it emits radiation with frequency $E_{m}-E_{n}=h v$, while the inverse transition can be induced if the electron absorbs radiation with the same frequency. A point which is not often emphasized is that, when he proposed his model, Bohr did not believe in light quanta, hence he did not phrase his postulates in terms of these entities.

\subsection{Step 6. Einstein 1917: Probability}

In 1916 [10], Einstein went back to the problem of cavity radiation, this time using insight coming from the Bohr theory. Unlike Bohr, Einstein explicitly assumed that in any transition, a single light quantum having energy equal to the energy difference is emitted or absorbed. Einstein considered radiation in thermal equilibrium with an ensemble of atoms or molecules, which therefore continuously absorb or emit light quanta. In fact, three kinds of elementary processes can occur, namely absorption, stimulated emission (in which a light quantum hits an atom, stimulating its decay to a lower energy state with emission of another quantum), and spontaneous emission (in which an atom spontaneously goes to a state with lower energy emitting a quantum). As the system is assumed to be at thermal equilibrium, the various states of the atoms will be distributed according to the Boltzmann distribution and, moreover, this distribution should not change with time, meaning that on average each transition from a state to another must happen with the same rate as the inverse transition. This translates in a detailed balance equation, from which the Planck distribution for the energy density of radiation follows. Two very important remarks are in order. First, the process of spontaneous emission is very different from the other two, as it is not triggered by the interaction with a light quantum. Instead, it seems to have no apparent cause, and it is necessary to assume that it occurs, in a given interval of time, with a given probability. Nor the direction in which the emerging light quantum is emitted is predictable. That is, spontaneous emission is an intrinsically probabilistic process, which seems to violate the principle of causality. This behavior is actually analogous to that of radioactive decay of a nucleus. Therefore, the Planck distribution can only be reproduced if intrinsically probabilistic and causality violating processes occur. This means that the quantum behaviour is inextricably linked with probability, a fact which is, of course, included in the full quantum mechanics, but which had not been anticipated.

\section{Results}

The above track was tested both with teachers and (in a reduced form for the moment) with selected students, and in the course of various outreach activities performed at the University of Salerno. The preliminary results are very encouraging. Teachers' responses also emphasized how they managed to see quantum physics from a new point of view, which helped them to overcome some criticalities in their understanding. Remarkably, also those whose major subject at university was physics shared this impression, as they did not encounter this way of treating the subject in their studies. The students found the material intriguing, not least due to the association with Einstein's name, and they developed some intuition about the quantum phenomena which were the object of study.

\section{Discussion}

As the majority of Einstein's fundamental ideas on light quanta came from statistical mechanics, of which he was a true master, it is possible to enhance our path by introducing the necessary tools of this subject, starting from the elementary notions of kinetic theory 
that are part of the standard curriculum. In particular, a knowledge of Boltzmann's entropy formula for the first part, and of Boltzmann's distribution of energies for the second part, is necessary. These tools can be introduced by again taking inspiration from Einstein's treatment, when available (in fact, he often added some review of the statistical tools he needed in his papers). Teaching elementary statistical physics in high schools is, however, a very active area of research on its own, with a large body of literature (see $[16,17])$, hence our proposal can also act as a link between the latter and the teaching of quantum physics. The possibility of using some of Einstein's papers in this endeavor as well has been the subject of a parallel study to the present one, and will be reported elsewhere.

Of course, the path developed in this paper does not constitute a complete course in quantum physics, for several reasons. First of all, relevant work by others still needs to be considered, of course. Moreover, the path entirely unfolds in the realm of the old quantum theory. After it is completed, an introduction to the basics of the full theory of quantum mechanics must be given. For this, a very rich literature has developed (see, e.g., [1] and references therein), hence there are various ways in which our proposal can be complemented. We have developed our own proposal in [18], again taking some inspiration from the heroic history of quantum mechanics. Preliminary tests of an enlarged didactic path involving a combination of both the proposals are beginning soon.

\title{
5. Conclusions
}

In this paper, we described a possible didactic path for elementary quantum physics, which, as part of the literature on the teaching of quantum physics, was inspired by the history of physics. The preliminary results we obtained indicate that this path can usefully complement already existing material, both in forming high school teachers, and in directly teaching students.

Author Contributions: The paper is the result of discussions between M.D.M., S.E. and A.N.; M.D.M. began to test parts of this proposals on students and teachers in the course of outreach activities performed at the University of Salerno. M.D.M. and A.N. analyzed the responses of students and teachers. M.D.M. wrote the paper. S.E. supervised the whole project. All authors have read and agreed to the published version of the manuscript.

Acknowledgments: The authors would like to thank A. Drago for suggesting in the first place that they investigate the pedagogical value of Einstein's 1905 paper.

Conflicts of Interest: The authors declare no conflict of interest.

\author{
Abbreviations \\ The following abbreviations are used in this manuscript: \\ RJ Rayleigh-Jeans
}

\section{References}

1. Krijtenburg-Lewerissa, K.; Pol, H.J.; Brinkman, A.; van Joolingen, W.R. Insights into teaching quantum mechanics in secondary and lower undergraduate education. Phys. Rev. Phys. Educ. Res. 2017, 13, 010109. Available online: https://journals.aps.org/ prper/abstract/10.1103/PhysRevPhysEducRes.13.010109 (accessed on 27 January 2021). [CrossRef]

2. Buongiorno, D.; Michelini, M. The conceptual contriibution of the history in learning physics: The case of optical spectroscopy. In Proceedings of the SISFA2017, Bari, Italy, 26-29 September 2017; Campanile, B., De Frenza, L., Garuccio, A., Eds.; Società Italiana degli Storici della Fisica e dell'Astronomia. Pavia University Press: Pavia, Italy, 2017; pp. 377-388.

3. Walker, J. Fundamentals of physics, 10th extended ed.; Wiley: Hoboke, NJ, USA, 2014.

4. $\quad$ Eisberg, R.E.; Resnick, R. Quantum Physics of Atoms, Molecules, Solids, Nuclei and Particles; Wiley: Hoboke, NJ, USA, 1985.

5. Bohm, D. Quantum Theory; Prentice-Hall: New York, NY, USA, 1951.

6. Messiah, A. Quantum Mechanics; Elsevier: Amsterdam, The Netherlands, 1961.

7. Pais, A. Subtle Is the Lord; Oxford University Press: Oxford, UK, 1982.

8. Einstein, A. Über einen die Erzeungung und Verwandlungndes Lichtes betreffenden heuristischen Gesichtspunkt. Ann. Phys. 1905, 17, 132-148. [CrossRef] 
9. Einstein, A. Entwicklung unserer Anschauungen über das Wesen und die Konstitution der Strahlung. Phys. Z. 1909, 10, 817-825. [CrossRef]

10. Einstein, A. Zur Quantentheorie der Strahlung. Phys. Z. 1917, 18, 121-128.

11. Duncan, A. The Conceptual Framework of Quantum Field Theory; Oxford University Press: Oxford, UK, 2012.

12. Paffuti, A. Note Sulla Nascita della Meccanica Quantistica; Pisa University Press: Pisa, Italy, 2013.

13. Cheng, T.-P. Einstein's Physics: Atoms, Quanta and Relativity Derived, Explained and Appraised; Oxford University Press: Oxford, UK, 2013.

14. Kennedy, R.E. A Student's Guide to Einstein's Major Papers; Oxford University Press: Oxford, UK, 2012.

15. The Collected Papers of Albert Einstein. Available online: https:/ / einsteinpapers.press.princeton.edu (accessed on 27 January 2021).

16. McDowell, S.A.C. A Simple Derivation of the Boltzmann Distribution. J. Chem. Ed. 1999, 76, 1393-1394. Available online: https:/ / pubs.acs.org/doi/abs/10.1021/ed076p1393 (accessed on 29 January 2021). [CrossRef]

17. Müller, R. The Boltzmann factor: A simplified derivation. Eur. J. Phys. 2014, 35, 055002. Available online: https://iopscience.iop. org/article/10.1088/0143-0807/35/5/055002 (accessed on 29 January 2021). [CrossRef]

18. Di Mauro, M.; Naddeo, A. Introducing quantum mechanics in high schools: A proposal based on Heisenberg's Umdeutung. In Proceedings of the 1st Electronic Conference on Universe, Online, 22-28 February 2021. 